PROCEEDINGS OF THE

AMERICAN MATHEMATICAL SOCIETY

Volume 140, Number 5, May 2012, Pages 1835-1847

S 0002-9939(2011)11049-9

Article electronically published on September 26, 2011

\title{
$k$-HARMONIC MAPS INTO A RIEMANNIAN MANIFOLD WITH CONSTANT SECTIONAL CURVATURE
}

\author{
SHUN MAETA
}

(Communicated by Jianguo Cao)

\begin{abstract}
J. Eells and L. Lemaire introduced $k$-harmonic maps, and Shaobo Wang showed the first variational formula. When $k=2$, it is called biharmonic maps (2-harmonic maps). There have been extensive studies in the area. In this paper, we consider the relationship between biharmonic maps and $k$-harmonic maps, and we show the non-existence theorem of 3-harmonic maps. We also give the definition of $k$-harmonic submanifolds of Euclidean spaces and study $k$-harmonic curves in Euclidean spaces. Furthermore, we give a conjecture for $k$-harmonic submanifolds of Euclidean spaces.
\end{abstract}

\section{INTRODUCTION}

The theory of harmonic maps has been applied to various fields in differential geometry. The harmonic maps between two Riemannian manifolds are critical maps of the energy functional $E(\phi)=\frac{1}{2} \int_{M}\|d \phi\|^{2} v_{g}$, for smooth maps $\phi: M \rightarrow N$.

On the other hand, in 1981, J. Eells and L. Lemaire [4] proposed the problem to consider the $k$-harmonic maps: they are critical maps of the functional

$$
E_{k}(\phi)=\int_{M} e_{k}(\phi) v_{g} \quad(k=1,2, \cdots),
$$

where $e_{k}(\phi)=\frac{1}{2}\left\|\left(d+d^{*}\right)^{k} \phi\right\|^{2}$ for smooth maps $\phi: M \rightarrow N$. G.Y. Jiang [6] studied the first and second variational formulas of the bi-energy $E_{2}$, and critical maps of $E_{2}$ are called biharmonic maps (2-harmonic maps). There have been extensive studies on biharmonic maps.

In 1989, Shaobo Wang [10] studied the first variational formula of the $k$-energy $E_{k}$, whose critical maps are called $k$-harmonic maps. Harmonic maps are always $k$-harmonic maps by definition. However, the author [7] showed biharmonic is not always $k$-harmonic $(k \geq 3)$. More generally, $s$-harmonic is not always $k$-harmonic $(s<k)$. Furthermore, the author [7] showed the second variational formula of the $k$-energy.

In this paper, we study $k$-harmonic maps into a Riemannian manifold with constant sectional curvature $K$.

In $\S 1$, we introduce notation and fundamental formulas of the tension field.

In $₫ 2$, we recall $k$-harmonic maps.

In 93 , we give the relationship between biharmonic maps and $k$-harmonic maps.

Received by the editors September 19, 2010 and, in revised form, January 20, 2011; January 27, 2001; and January 29, 2011.

2010 Mathematics Subject Classification. Primary 58E20; Secondary 53C43.

(C)2011 American Mathematical Society 1835 
In 4 we study 3-harmonic maps into a non-positive sectional curvature and obtain a non-existence theorem.

Finally, in $\$ 5$ we define $k$-harmonic submanifolds of Euclidean spaces. Also, we show that a $k$-harmonic curve is a straight line. Furthermore, we give a conjecture for $k$-harmonic submanifolds in Euclidean spaces.

\section{Preliminaries}

Let $(M, g)$ be an $m$ dimensional Riemannian manifold, $(N, h)$ an $n$ dimensional one, and $\phi: M \rightarrow N$ a smooth map. We use the following notation.

The second fundamental form $B(\phi)$ of $\phi$ is a covariant differentiation $\widetilde{\nabla} d \phi$ of the 1-form $d \phi$, which is a section of $\odot^{2} T^{*} M \otimes \phi^{-1} T N$. For every $X, Y \in \Gamma(T M)$, let

$$
\begin{aligned}
B(X, Y) & =(\widetilde{\nabla} d \phi)(X, Y)=\left(\widetilde{\nabla}_{X} d \phi\right)(Y) \\
& =\bar{\nabla}_{X} d \phi(Y)-d \phi\left(\nabla_{X} Y\right)=\nabla_{d \phi(X)}^{N} d \phi(Y)-d \phi\left(\nabla_{X} Y\right) .
\end{aligned}
$$

Here, $\nabla, \nabla^{N}, \bar{\nabla}, \widetilde{\nabla}$ are the induced connections on the bundles $T M, T N, \phi^{-1} T N$ and $T^{*} M \otimes \phi^{-1} T N$, respectively.

If $M$ is compact, we consider critical maps of the energy functional

$$
E(\phi)=\int_{M} e(\phi) v_{g}
$$

where $e(\phi)=\frac{1}{2}\|d \phi\|^{2}=\sum_{i=1}^{m} \frac{1}{2}\left\langle d \phi\left(e_{i}\right), d \phi\left(e_{i}\right)\right\rangle$, which is called the energy density of $\phi$, the inner product $\langle\cdot, \cdot\rangle$ is a Riemannian metric $h$, and $\left\{e_{i}\right\}_{i=1}^{m}$ is a locally defined orthonormal frame field on $(M, g)$. The tension field $\tau(\phi)$ of $\phi$ is defined by

$$
\tau(\phi)=\sum_{i=1}^{m}(\widetilde{\nabla} d \phi)\left(e_{i}, e_{i}\right)=\sum_{i=1}^{m}\left(\widetilde{\nabla}_{e_{i}} d \phi\right)\left(e_{i}\right)
$$

Then, $\phi$ is a harmonic map if $\tau(\phi)=0$.

The curvature tensor field $R^{N}(\cdot, \cdot)$ of the Riemannian metric on the bundle $T N$ is defined as follows:

$$
R^{N}(X, Y)=\nabla_{X}^{N} \nabla_{Y}^{N}-\nabla_{Y}^{N} \nabla_{X}^{N}-\nabla_{[X, Y]}^{N} \quad(X, Y \in \Gamma(T N)) .
$$

$\bar{\triangle}=\bar{\nabla}^{*} \bar{\nabla}=-\sum_{k=1}^{m}\left(\bar{\nabla}_{e_{k}} \bar{\nabla}_{e_{k}}-\bar{\nabla}_{\nabla_{e_{k} e_{k}}}\right)$ is the rough Laplacian.

Also, Jiang [6] showed that $\phi:(M, g) \rightarrow(N, h)$ is a biharmonic (2-harmonic) if and only if

$$
\bar{\triangle} \tau(\phi)-\sum_{i=1}^{m} R^{N}\left(\tau(\phi), d \phi\left(e_{i}\right)\right) d \phi\left(e_{i}\right)=0 .
$$

Throughout the paper, we omit the sign $\sum$ without mentioning this omission.

\section{2. $k$-HARMONIC MAPS}

J. Eells and L. Lemaire [4] proposed the notation of $k$-harmonic maps. The Euler-Lagrange equations for the $k$-harmonic maps were shown by Shaobo Wang [10. In this section, we recall $k$-harmonic maps. 
We consider a smooth variation $\left\{\phi_{t}\right\}_{t \in I_{\epsilon}}\left(I_{\epsilon}=(-\epsilon, \epsilon)\right)$ of $\phi$ with parameters $t$; i.e. we consider the smooth map $F$ given by

$$
F: I_{\epsilon} \times M \rightarrow N, F(t, p)=\phi_{t}(p)
$$

where $F(0, p)=\phi_{0}(p)=\phi(p)$, for all $p \in M$.

The corresponding variational vector field $V$ is given by

$$
V(p)=\left.\frac{d}{d t}\right|_{t=0} \phi_{t, 0} \in T_{\phi(p)} N,
$$

where $V$ is a section of $\phi^{-1} T N$, i.e. $V \in \Gamma\left(\phi^{-1} T N\right)$.

We also denote by $\nabla, \bar{\nabla}$ and $\widetilde{\nabla}$ the induced Riemannian connection on $T\left(I_{\epsilon} \times M\right)$, $F^{-1} T N$ and $T^{*}\left(I_{\epsilon} \times M\right) \otimes F^{-1} T N$, respectively.

Definition 2.1 (4]). For $k=1,2, \cdots$ the $k$-energy functional is defined by

$$
E_{k}(\phi)=\frac{1}{2} \int_{M}\left\|\left(d+d^{*}\right)^{k} \phi\right\|^{2} v_{g}, \quad \phi \in C^{\infty}(M, N) .
$$

Then, $\phi$ is $k$-harmonic if it is a critical point of $E_{k}$; i.e., for all smooth variations $\left\{\phi_{t}\right\}$ of $\phi$ with $\phi_{0}=\phi$,

$$
\left.\frac{d}{d t}\right|_{t=0} E_{k}\left(\phi_{t}\right)=0
$$

We say that a $k$-harmonic map is proper if it is not harmonic.

Lemma $2.2([10])$.

$$
\begin{aligned}
&\left.\bar{\nabla}_{\frac{\partial}{\partial t}} \bar{\triangle}^{s-1} \tau(F)\right|_{t=0}=- \bar{\triangle}^{s} V+\bar{\triangle}^{s-1} R^{N}\left(V, d \phi\left(e_{j}\right)\right) d \phi\left(e_{j}\right) \\
&+\sum_{l=1}^{s-1} \bar{\triangle}^{l-1}\left\{-\bar{\nabla}_{e_{j}} R^{N}\left(V, d \phi\left(e_{j}\right)\right) \bar{\triangle}^{s-l-1} \tau(\phi)\right. \\
&-R^{N}\left(V, d \phi\left(e_{j}\right)\right) \bar{\nabla}_{e_{j}} \bar{\triangle}^{s-l-1} \tau(\phi) \\
& \\
&\left.+R^{N}\left(V, d \phi\left(\nabla_{e_{j}} e_{j}\right)\right) \bar{\triangle}^{s-l-1} \tau(\phi)\right\} .
\end{aligned}
$$

Proof. For all $\omega \in \Gamma\left(\phi^{-1} T N\right)$,

$$
\begin{aligned}
\bar{\nabla}_{\frac{\partial}{\partial t}} \bar{\triangle} \omega= & -\left\{\bar{\nabla}_{\frac{\partial}{\partial t}}\left(\bar{\nabla}_{e_{j}} \bar{\nabla}_{e_{j}}-\bar{\nabla}_{\nabla_{e_{j} e_{j}}}\right) \omega\right\} \\
= & -\left\{\bar{\nabla}_{e_{j}} \bar{\nabla}_{\frac{\partial}{\partial t}}\left(\bar{\nabla}_{e_{j}} \omega\right)+R^{N}\left(d F\left(\frac{\partial}{\partial t}\right), d F\left(e_{j}\right)\right) \bar{\nabla}_{e_{j}} \omega\right. \\
& \left.-\bar{\nabla}_{\nabla_{e_{j}} e_{j}} \bar{\nabla}_{\frac{\partial}{\partial t}} \omega-R^{N}\left(d F\left(\frac{\partial}{\partial t}\right), d F\left(\nabla_{e_{j}} e_{j}\right)\right) \omega\right\} \\
= & -\left\{\bar{\nabla}_{e_{j}}\left(\bar{\nabla}_{e_{j}} \bar{\nabla}_{\frac{\partial}{\partial t}} \omega+R^{N}\left(d F\left(\frac{\partial}{\partial t}\right), d F\left(e_{j}\right)\right) \omega\right)\right. \\
& +R^{N}\left(d F\left(\frac{\partial}{\partial t}\right), d F\left(e_{j}\right)\right) \bar{\nabla}_{e_{j}} \omega \\
& \left.-\bar{\nabla}_{\nabla_{e_{j}} e_{j}} \bar{\nabla}_{\frac{\partial}{\partial t}} \omega-R^{N}\left(d F\left(\frac{\partial}{\partial t}\right), d F\left(\nabla_{e_{j}} e_{j}\right)\right) \omega\right\} .
\end{aligned}
$$

Repeating this and using

$$
\left.\bar{\nabla}_{\frac{\partial}{\partial t}} \tau(F)\right|_{t=0}=-\bar{\triangle} V+R^{N}\left(V, d \phi\left(e_{j}\right)\right) d \phi\left(e_{j}\right)
$$

we have the lemma. 
Lemma 2.3 ([10]).

$$
\begin{aligned}
\left.\bar{\nabla}_{\frac{\partial}{\partial t}} \bar{\nabla}_{e_{i}} \bar{\triangle}^{s-1} \tau(F)\right|_{t=0}= & -\bar{\nabla}_{e_{i}} \bar{\triangle}^{s} V+\bar{\nabla}_{e_{i}} \bar{\triangle}^{s-1} R^{N}\left(V, d \phi\left(e_{j}\right)\right) d \phi\left(e_{j}\right) \\
& +\sum_{l=1}^{s-1} \bar{\nabla}_{e_{i}} \bar{\triangle}^{l-1}\left\{-\bar{\nabla}_{e_{j}} R^{N}\left(V, d \phi\left(e_{j}\right)\right) \bar{\triangle}^{s-l-1} \tau(\phi)\right. \\
& -R^{N}\left(V, d \phi\left(e_{j}\right)\right) \bar{\nabla}_{e_{j}} \bar{\triangle}^{s-l-1} \tau(\phi) \\
& \left.+R^{N}\left(V, d \phi\left(\nabla_{e_{j}} e_{j}\right)\right) \bar{\triangle}^{s-l-1} \tau(\phi)\right\} \\
& +R^{N}\left(V, d \phi\left(e_{i}\right)\right) \bar{\triangle}^{s-1} \tau(\phi) .
\end{aligned}
$$

Proof.

$$
\bar{\nabla}_{\frac{\partial}{\partial t}} \bar{\nabla}_{e_{i}} \bar{\triangle}^{s-1} \tau(F)=\bar{\nabla}_{e_{i}} \bar{\nabla}_{\frac{\partial}{\partial t}} \bar{\triangle}^{s-1} \tau(F)+R^{N}\left(d F\left(\frac{\partial}{\partial t}\right), d F\left(e_{i}\right)\right) \bar{\triangle}^{s-1} \tau(F) .
$$

Using Lemma 2.2. we have the lemma.

Lemma 2.4 ([10]).

$$
\begin{array}{r}
\int_{M}\left\langle\bar{\nabla}_{e_{j}} R^{N}\left(V, d \phi\left(e_{j}\right)\right) V_{1}-R^{N}\left(V, d \phi\left(\nabla_{e_{j}} e_{j}\right)\right) V_{1}, V_{2}\right\rangle v_{g} \\
=-\int_{M}\left\langle R^{N}\left(V, d \phi\left(e_{j}\right)\right) V_{1}, \bar{\nabla}_{e_{j}} V_{2}\right\rangle v_{g}
\end{array}
$$

$V_{1}, V_{2} \in \Gamma\left(\phi^{-1} T N\right)$.

Proof.

$$
\begin{aligned}
\operatorname{div}\left(\left\langle R^{N}\left(V, d \phi\left(e_{i}\right)\right) V_{1}, V_{2}\right\rangle e_{i}\right)= & \left\langle\nabla_{e_{j}}\left\langle R^{N}\left(V, d \phi\left(e_{i}\right)\right) V_{1}, V_{2}\right\rangle e_{i}, e_{j}\right\rangle \\
= & \left\langle\left\langle\bar{\nabla}_{e_{j}} R^{N}\left(V, d \phi\left(e_{i}\right)\right) V_{1}, V_{2}\right\rangle e_{i}\right. \\
& +\left\langle R^{N}\left(V, d \phi\left(e_{i}\right)\right) V_{1}, \bar{\nabla}_{e_{j}} V_{2}\right\rangle e_{i} \\
& \left.+\left\langle R^{N}\left(V, d \phi\left(e_{i}\right)\right) V_{1}, V_{2}\right\rangle \nabla_{e_{j}} e_{i}, e_{j}\right\rangle .
\end{aligned}
$$

By Green's theorem, we have

$$
\begin{aligned}
0= & \int_{M} \operatorname{div}\left\langle R^{N}\left(V, d \phi\left(e_{i}\right)\right) V_{1}, V_{2}\right\rangle e_{i} v_{g} \\
= & \int_{M}\left\langle\bar{\nabla}_{e_{j}} R^{N}\left(V, d \phi\left(e_{i}\right) V_{1}, V_{2}\right)\right\rangle \delta_{i j} \\
& +\left\langle R^{N}\left(V, d \phi\left(e_{i}\right)\right) V_{1}, \bar{\nabla}_{e_{j}} V_{2}\right\rangle \delta_{i j} \\
& +\left\langle R^{N}\left(V, d \phi\left(e_{i}\right)\right) V_{1}, V_{2}\right\rangle\left\langle\nabla_{e_{j}} e_{i}, e_{j}\right\rangle v_{g} .
\end{aligned}
$$

Here,

$$
\begin{aligned}
\left\langle R^{N}\left(V, d \phi\left(e_{i}\right)\right) V_{1}, V_{2}\right\rangle\left\langle\nabla_{e_{j}} e_{i}, e_{j}\right\rangle & =\left\langle R^{N}\left(V, d \phi\left(\left\langle\nabla_{e_{j}} e_{i}, e_{j}\right\rangle e_{i}\right)\right) V_{1}, V_{2}\right\rangle \\
& =-\left\langle R^{N}\left(V, d \phi\left(\nabla_{e_{i}} e_{i}\right)\right) V_{1}, V_{2}\right\rangle .
\end{aligned}
$$

Therefore, we have the lemma. 
Theorem 2.5 ([10]). Let $k=2 s(s=1,2, \cdots)$,

$$
\left.\frac{d}{d t}\right|_{t=0} E_{2 s}\left(\phi_{t}\right)=-\int_{M}\left\langle\tau_{2 s}(\phi), V\right\rangle,
$$

where

$$
\begin{aligned}
\tau_{2 s}(\phi)=\bar{\triangle}^{2 s-1} \tau(\phi)-R^{N}\left(\bar{\triangle}^{2 s-2} \tau(\phi), d \phi\left(e_{j}\right)\right) e \phi\left(e_{j}\right) \\
-\sum_{l=1}^{s-1}\left\{R^{N}\left(\bar{\nabla}_{e_{j}} \bar{\triangle}^{s+l-2} \tau(\phi), \bar{\triangle}^{s-l-1} \tau(\phi)\right) d \phi\left(e_{j}\right)\right. \\
\left.-R^{N}\left(\bar{\triangle}^{s+l-2} \tau(\phi), \bar{\nabla}_{e_{j}} \bar{\triangle}^{s-l-1} \tau(\phi)\right) d \phi\left(e_{j}\right)\right\},
\end{aligned}
$$

where $\bar{\triangle}^{-1}=0$.

Proof.

$$
\begin{aligned}
E_{2 s}(\phi) & =\int_{M}\langle\underbrace{\left\langle\left(d^{*} d\right) \cdots\left(d^{*} d\right)\right.}_{s} \phi, \underbrace{\left(d^{*} d\right) \cdots\left(d^{*} d\right)}_{s} \phi\rangle v_{g} \\
& =\int_{M}\left\langle\bar{\triangle}^{s-1} \tau(\phi), \bar{\triangle}^{s-1} \tau(\phi)\right\rangle v_{g} .
\end{aligned}
$$

By using Lemma 2.2 and Lemma 2.4, we calculate $\frac{d}{d t} E_{2 s}\left(\phi_{t}\right)$ :

$$
\begin{aligned}
& \left.\quad \frac{d}{d t} E_{2 s}\left(\phi_{t}\right)\right|_{t=0} \\
& =\left.\int_{M}\left\langle\bar{\nabla}_{\frac{\partial}{\partial t}} \bar{\triangle}^{s-1} \tau(F), \bar{\triangle}^{s-1} \tau(F)\right\rangle v_{g}\right|_{t=0} \\
& =\int_{M}\left\langle-\bar{\triangle}^{s} V+\bar{\triangle}^{s-1} R^{N}\left(V, d \phi\left(e_{j}\right)\right) d \phi\left(e_{j}\right)\right. \\
& \quad+\sum_{l=1}^{s-1} \bar{\triangle}^{l-1}\left\{-\bar{\nabla}_{e_{j}} R^{N}\left(V, d \phi\left(e_{j}\right)\right) \bar{\triangle}^{s-l-1} \tau(\phi)\right. \\
& \quad-R^{N}\left(V, d \phi\left(e_{j}\right)\right) \bar{\nabla}_{e_{j}} \bar{\triangle}^{s-l-1} \tau(\phi) \\
& \left.\left.\quad+R^{N}\left(V, d \phi\left(\nabla_{e_{j}} e_{j}\right)\right) \bar{\triangle}^{s-l-1} \tau(\phi), \bar{\triangle}^{s-1} \tau(\phi)\right\}\right\rangle \\
& =\int_{M}\left\langle V,-\bar{\triangle}^{2 s-1} \tau(\phi)\right\rangle v_{g} \\
& +\int_{M}\left\langle V, R^{N}\left(\bar{\triangle}^{2 s-2} \tau(\phi), d \phi\left(e_{j}\right)\right) d \phi\left(e_{j}\right)\right\rangle v_{g} \\
& +\sum_{l=1}^{s-1} \int_{M}\left\langle-\bar{\nabla}_{e_{j}} R^{N}\left(V, d \phi\left(e_{j}\right)\right) \bar{\triangle}^{s-l-1} \tau(\phi)\right. \\
& \quad-R^{N}\left(V, d \phi\left(e_{j}\right)\right) \bar{\nabla}_{e_{j}} \bar{\triangle}^{s-l-1} \tau(\phi) \\
& \left.\quad+R^{N}\left(V, d \phi\left(\nabla_{e_{j}} e_{j}\right)\right) \bar{\triangle}^{s-l-1} \tau(\phi), \bar{\triangle}^{s+l-2} \tau(\phi)\right\rangle v_{g}
\end{aligned}
$$




$$
\begin{aligned}
= & \int_{M}\left\langle V,-\bar{\triangle}^{2 s-1} \tau(\phi)\right\rangle v_{g} \\
& +\int_{M}\left\langle V, R^{N}\left(\bar{\triangle}^{2 s-2} \tau(\phi), d \phi\left(e_{j}\right)\right) d \phi\left(e_{j}\right)\right\rangle v_{g} \\
& +\sum_{l=1}^{s-1}\left\{\int_{M}\left\langle R^{N}\left(V, d \phi\left(e_{j}\right)\right) \bar{\triangle}^{s-l-1} \tau(\phi), \bar{\nabla}_{e_{j}} \bar{\triangle}^{s+l-2} \tau(\phi)\right\rangle v_{g}\right. \\
& \left.+\int_{M}\left\langle-R^{N}\left(V, d \phi\left(e_{j}\right)\right) \bar{\nabla}_{e_{j}} \bar{\triangle}^{s-l-1} \tau(\phi), \bar{\triangle}^{s+l-2} \tau(\phi)\right\rangle v_{g}\right\} \\
= & \int_{M}\left\langle V,-\bar{\triangle}^{2 s-1} \tau(\phi)\right\rangle v_{g} \\
& +\int_{M}\left\langle V, R^{N}\left(\bar{\triangle}^{2 s-2} \tau(\phi), d \phi\left(e_{j}\right)\right) d \phi\left(e_{j}\right)\right\rangle v_{g} \\
& +\sum_{l=1}^{s-1}\left\{\int_{M}\left\langle R^{N}\left(\bar{\nabla}_{e_{j}} \bar{\triangle}^{s+l-2} \tau(\phi), \bar{\triangle}^{s-l-1} \tau(\phi)\right) d \phi\left(e_{j}\right), V\right\rangle v_{g}\right. \\
& \left.-\int_{M}\left\langle R^{N}\left(\bar{\triangle}^{s+l-2} \tau(\phi), \bar{\nabla}_{e_{j}} \bar{\triangle}^{s-l-1} \tau(\phi)\right) d \phi\left(e_{j}\right), V\right\rangle v_{g}\right\} \\
= & \int_{M}\left\langle V,-\bar{\triangle}^{2 s-1} \tau(\phi)+R^{N}\left(\bar{\triangle}^{2 s-2} \tau(\phi), d \phi\left(e_{j}\right)\right) d \phi\left(e_{j}\right)\right. \\
& +\sum_{l=1}^{s-1}\left\{R^{N}\left(\bar{\nabla}_{e_{j}} \bar{\triangle}^{s+l-2} \tau(\phi), \bar{\triangle}^{s-l-1} \tau(\phi)\right) d \phi\left(e_{j}\right)\right. \\
& \left.\left.-R^{N}\left(\bar{\triangle}^{s+l-2} \tau(\phi), \bar{\nabla}_{e_{j}} \bar{\triangle}^{s-l-1} \tau(\phi)\right) d \phi\left(e_{j}\right)\right\}\right\rangle v_{g} .
\end{aligned}
$$

Therefore, we have the theorem.

Theorem 2.6 ([10]). Let $k=2 s+1 \quad(s=0,1,2, \cdots)$,

$$
\left.\frac{d}{d t}\right|_{t=0} E_{2 s+1}\left(\phi_{t}\right)=-\int_{M}\left\langle\tau_{2 s+1}(\phi), V\right\rangle,
$$

where

$$
\begin{aligned}
\tau_{2 s+1}(\phi)= & \bar{\triangle}^{2 s} \tau(\phi)-R^{N}\left(\bar{\triangle}^{2 s-1} \tau(\phi), d \phi\left(e_{j}\right)\right) d \phi\left(e_{j}\right) \\
& -\sum_{l=1}^{s-1}\left\{R^{N}\left(\bar{\nabla}_{e_{j}} \bar{\triangle}^{s+l-1} \tau(\phi), \bar{\triangle}^{s-l-1} \tau(\phi)\right) d \phi\left(e_{j}\right)\right. \\
& \left.-R^{N}\left(\bar{\triangle}^{s+l-1} \tau(\phi), \bar{\nabla}_{e_{j}} \bar{\triangle}^{s-l-1} \tau(\phi)\right) d \phi\left(e_{j}\right)\right\} \\
& -R^{N}\left(\bar{\nabla}_{e_{j}} \bar{\triangle}^{s-1} \tau(\phi), \bar{\triangle}^{s-1} \tau(\phi)\right) d \phi\left(e_{j}\right),
\end{aligned}
$$

where $\bar{\triangle}^{-1}=0$.

Proof. When $s=0$, it is a well-known harmonic map. Hence we consider the case of $s=1,2, \cdots$ :

$$
\begin{aligned}
E_{2 s+1}(\phi) & =\int_{M}\langle d \underbrace{\left(d^{*} d\right) \cdots\left(d^{*} d\right)}_{s} \phi, d \underbrace{\left(d^{*} d\right) \cdots\left(d^{*} d\right)}_{s} \phi\rangle v_{g} \\
& =\int_{M}\left\langle\bar{\nabla}_{e_{i}} \bar{\triangle}^{s-1} \tau(\phi), \bar{\nabla}_{e_{i}} \bar{\triangle}^{s-1} \tau(\phi)\right\rangle v_{g} .
\end{aligned}
$$


By using Lemma 2.3 and Lemma 2.4, we calculate $\frac{d}{d t} E_{2 s+1}\left(\phi_{t}\right)$ :

$$
\begin{aligned}
& \left.\quad \frac{d}{d t} E_{2 s+1}\left(\phi_{t}\right)\right|_{t=0} \\
& =\left.\int_{M}\left\langle\bar{\nabla}_{\frac{\partial}{\partial t}} \bar{\nabla}_{e_{i}} \bar{\triangle}^{s-1} \tau(F), \bar{\nabla}_{e_{i}} \bar{\triangle}^{s-1} \tau(F)\right\rangle v_{g}\right|_{t=0} \\
& =\int_{M}\left\langle-\bar{\nabla}_{e_{i}} \bar{\triangle}^{s} V+\bar{\nabla}_{e_{i}} \bar{\triangle}^{s-1} R^{N}\left(V, d \phi\left(e_{j}\right)\right) d \phi\left(e_{j}\right)\right. \\
& +\sum_{l=1}^{s-1} \bar{\nabla}_{e_{i}} \bar{\triangle}^{l-1}\left\{-\bar{\nabla}_{e_{j}} R^{N}\left(V, d \phi\left(e_{j}\right)\right) \bar{\triangle}^{s-l-1} \tau(\phi)\right. \\
& \quad-R^{N}\left(V, d \phi\left(e_{j}\right)\right) \bar{\nabla}_{e_{j}} \bar{\triangle}^{s-l-1} \tau(\phi) \\
& +R^{N}\left(V, d \phi\left(\nabla_{e_{j}} e_{j}\right)\right) \bar{\triangle}^{s-l-1} \tau(\phi) \\
& \left.\left.+R^{N}\left(V, d \phi\left(e_{i}\right)\right) \bar{\triangle}^{s-1} \tau(\phi)\right\}, \bar{\nabla}_{e_{i}} \bar{\triangle}^{s-1} \tau(\phi)\right\rangle v_{g} .
\end{aligned}
$$

Here, using

$$
\int_{M}\left\langle\bar{\nabla}_{e_{i}} \omega_{1}, \bar{\nabla}_{e_{i}} \omega_{2}\right\rangle v_{g}=\int_{M}\left\langle\bar{\triangle} \omega_{1}, \omega_{2}\right\rangle v_{g}
$$

where $\omega_{1}, \omega_{2} \in \Gamma\left(\phi^{-1} T N\right)$, we have

$$
\begin{aligned}
& \left.\quad \frac{d}{d t} E_{2 s+1}\left(\phi_{t}\right)\right|_{t=0} \\
& =\int_{M}\left\langle V,-\bar{\triangle}^{2 s} \tau(\phi)\right\rangle v_{g} \\
& \quad+\int_{M}\left\langle R^{N}\left(V, d \phi\left(e_{j}\right)\right) d \phi\left(e_{j}\right), \bar{\triangle}^{2 s-1} \tau(\phi)\right\rangle v_{g} \\
& \quad+\sum_{l=1}^{s-1} \int_{M}\left\langle-\bar{\nabla}_{e_{j}} R^{N}\left(V, d \phi\left(e_{j}\right)\right) \bar{\triangle}^{s-l-1} \tau(\phi)\right. \\
& \quad-R^{N}\left(V, d \phi\left(e_{j}\right)\right) \bar{\nabla}_{e_{j}} \bar{\triangle}^{s-l-1} \tau(\phi) \\
& \left.\quad+R^{N}\left(V, d \phi\left(\nabla_{e_{j}} e_{j}\right)\right) \bar{\triangle}^{s-l-1} \tau(\phi), \bar{\triangle}^{s+l-1} \tau(\phi)\right\rangle v_{g} \\
& \quad+\int_{M}\left\langle R^{N}\left(V, d \phi\left(e_{i}\right)\right) \bar{\triangle}^{s-1} \tau(\phi), \bar{\nabla}_{e_{i}} \bar{\triangle}^{s-1} \tau(\phi)\right\rangle v_{g}
\end{aligned}
$$




$$
\begin{gathered}
=\int_{M}\left\langle V,-\bar{\triangle}^{2 s} \tau(\phi)+R^{N}\left(\bar{\triangle}^{2 s-1} \tau(\phi), d \phi\left(e_{j}\right)\right) d \phi\left(e_{j}\right)\right. \\
+\sum_{l=1}^{s-1}\left\{R^{N}\left(\bar{\nabla}_{e_{j}} \bar{\triangle}^{s+l-1} \tau(\phi), \bar{\triangle}^{s-l-1} \tau(\phi)\right) d \phi\left(e_{j}\right)\right. \\
\left.\quad-R^{N}\left(\bar{\triangle}^{s+l-1} \tau(\phi), \bar{\nabla}_{e_{j}} \bar{\triangle}^{s-l-1} \tau(\phi)\right) d \phi\left(e_{j}\right)\right\} \\
\left.+R^{N}\left(\bar{\nabla}_{e_{j}} \bar{\triangle}^{s-1} \tau(\phi), \bar{\triangle}^{s-1} \tau(\phi)\right) d \phi\left(e_{j}\right)\right\rangle v_{g} .
\end{gathered}
$$

Therefore, we have the theorem.

By Theorems 2.5 and 2.6, we have the following [10].

Corollary 2.7. A harmonic map is always a $k$-harmonic map $(k=1,2, \cdots)$.

\section{The RELATIONSHIP BETWEen BIHARMONIC AND $k$-HARMONIC}

In [7, the author showed that $s$-harmonic is not always $k$-harmonic $(s<k)$. Especially, biharmonic is not always $k$-harmonic $(k \geq 3)$. Therefore, we study the relationship between biharmonic and $k$-harmonic $(2<k)$. We obtain some results.

Proposition 3.1. Let $\phi:(M, g) \rightarrow(N, h)$ be an isometric immersion into a Riemannian manifold with constant sectional curvature $K$. Then $\phi$ is biharmonic if and only if

$$
\bar{\triangle} \tau(\phi)=K m \tau(\phi)
$$

Proof. $\phi$ is biharmonic if and only if

$$
\begin{aligned}
0 & =\bar{\triangle} \tau(\phi)-R^{N}\left(\tau(\phi), d \phi\left(e_{i}\right)\right) d \phi\left(e_{i}\right) \\
& =\bar{\triangle} \tau(\phi)-K\left\{\left\langle d \phi\left(e_{i}\right), d \phi\left(e_{i}\right)\right\rangle \tau(\phi)-\left\langle d \phi\left(e_{i}\right), \tau(\phi)\right\rangle d \phi\left(e_{i}\right)\right\} \\
& =\bar{\triangle} \tau(\phi)-K m \tau(\phi) .
\end{aligned}
$$

Thus, we have the proposition.

Lemma 3.2. Let $\phi:(M, g) \rightarrow(N, h)$ be an isometric immersion into a Riemannian manifold with constant sectional curvature $K$. If $\phi$ is biharmonic,

$$
\left\langle d \phi\left(e_{i}\right), \bar{\triangle}^{l} \tau(\phi)\right\rangle=0 \quad(l=0,1, \cdots) .
$$

Proof. By using Proposition 3.1, we have

$$
\begin{aligned}
\left\langle d \phi\left(e_{i}\right), \bar{\triangle}^{l} \tau(\phi)\right\rangle & =m K\left\langle d \phi\left(e_{i}\right), \bar{\triangle}^{l-1} \tau(\phi)\right\rangle \\
& \cdots \\
& =(m K)^{l}\left\langle d \phi\left(e_{i}\right), \tau(\phi)\right\rangle \\
& =0 .
\end{aligned}
$$

Lemma 3.3. Let $\phi:(M, g) \rightarrow(N, h)$ be an isometric immersion into a Riemannian manifold with constant sectional curvature $K$. If $\phi$ is biharmonic,

$$
\left\langle d \phi\left(e_{i}\right), \bar{\nabla}_{e_{i}} \bar{\triangle}^{l} \tau(\phi)\right\rangle=-(m K)^{l}\|\tau(\phi)\|^{2} .
$$


Proof. By using Proposition 3.1, we have

$$
\begin{aligned}
\left\langle d \phi\left(e_{i}\right), \bar{\nabla}_{e_{i}} \bar{\triangle}^{l} \tau(\phi)\right\rangle & =m K\left\langle d \phi\left(e_{i}\right), \bar{\nabla}_{e_{i}} \bar{\triangle}^{l-1} \tau(\phi)\right\rangle \\
& \cdots \\
& =(m K)^{l}\left\langle d \phi\left(e_{i}\right), \bar{\nabla}_{e_{i}} \tau(\phi)\right\rangle \\
& =-(m K)^{l}\|\tau(\phi)\|^{2}
\end{aligned}
$$

where, in the last equation, we only notice that

$$
0=e_{i}\left\langle d \phi\left(e_{i}\right), \tau(\phi)\right\rangle=\left\langle\bar{\nabla}_{e_{i}} d \phi\left(e_{i}\right), \tau(\phi)\right\rangle+\left\langle d \phi\left(e_{i}\right), \bar{\nabla}_{e_{i}} \tau(\phi)\right\rangle .
$$

Using these lemmas, we show the following two theorems.

Theorem 3.4. Let $\phi:(M, g) \rightarrow(N, h)$ be a biharmonic isometric immersion into a Riemannian manifold with constant sectional curvature $K(\neq 0)$. If $\phi$ is $2 s$-harmonic $(s \geq 2)$, $\phi$ is harmonic.

Proof. By Theorem 2.5, $\phi$ is $2 s$-harmonic if and only if

$$
\begin{aligned}
& \bar{\triangle}^{2 s-1} \tau(\phi)-K\left\{m \bar{\triangle}^{2 s-2} \tau(\phi)-\left\langle d \phi\left(e_{j}\right), \bar{\triangle}^{2 s-2} \tau(\phi)\right\rangle d \phi\left(e_{j}\right)\right\} \\
&-\sum_{l=1}^{s-1}\{ K\left(\left\langle\bar{\triangle}^{s-l-1} \tau(\phi), d \phi\left(e_{j}\right)\right\rangle \bar{\nabla}_{e_{j}} \bar{\triangle}^{s+l-2} \tau(\phi)\right. \\
&-\left\langle d \phi\left(e_{j}\right), \bar{\nabla}_{e_{j}} \bar{\triangle}^{s+l-2} \tau(\phi)\right\rangle \bar{\triangle}^{s-l-1} \tau(\phi) \\
&-\left\langle\bar{\nabla}_{e_{j}} \bar{\triangle}^{s-l-1} \tau(\phi), d \phi\left(e_{j}\right)\right\rangle \bar{\triangle}^{s+l-2} \tau(\phi) \\
&\left.\left.+\left\langle d \phi\left(e_{j}\right), \bar{\triangle}^{s+l-2} \tau(\phi)\right\rangle \bar{\nabla}_{e_{j}} \bar{\triangle}^{s-l-1} \tau(\phi)\right)\right\}=0 .
\end{aligned}
$$

By Proposition 3.1 and Lemmas 3.2 and 3.3 , we have

$$
\begin{aligned}
0= & (m K)^{2 s-1} \tau(\phi)-(m K)^{2 s-1} \tau(\phi) \\
& -\sum_{l=1}^{s-1}\left\{K\left((m K)^{2 s-3}\|\tau(\phi)\|^{2} \tau(\phi)+(m K)^{2 s-3}\|\tau(\phi)\|^{2} \tau(\phi)\right)\right\} \\
= & -2(s-1) K(m K)^{2 s-3}\|\tau(\phi)\|^{2} \tau(\phi) .
\end{aligned}
$$

Thus, we have the theorem.

Theorem 3.5. Let $\phi:(M, g) \rightarrow(N, h)$ be a biharmonic isometric immersion into a Riemannian manifold with constant sectional curvature $K(\neq 0)$. If $\phi$ is $(2 s+1)$-harmonic $(s \geq 1)$, $\phi$ is harmonic. 
Proof. By Theorem 2.6. $\phi$ is $(2 s+1)$-harmonic if and only if

$$
\begin{aligned}
\bar{\triangle}^{2 s} \tau(\phi) & -K\left\{m \bar{\triangle}^{2 s-1} \tau(\phi)-\left\langle d \phi\left(e_{j}\right), \bar{\triangle}^{2 s-1} \tau(\phi)\right\rangle d \phi\left(e_{j}\right)\right\} \\
-\sum_{l=1}^{s-1}\{ & K\left(\left\langle\bar{\triangle}^{s-l-1} \tau(\phi), d \phi\left(e_{j}\right)\right\rangle \bar{\nabla}_{e_{j}} \bar{\triangle}^{s+l-1} \tau(\phi)\right. \\
& -\left\langle d \phi\left(e_{j}\right), \bar{\nabla}_{e_{j}} \bar{\triangle}^{s+l-1} \tau(\phi)\right\rangle \bar{\triangle}^{s-l-1} \tau(\phi) \\
& -\left\langle\bar{\nabla}_{e_{j}} \bar{\triangle}^{s-l-1} \tau(\phi), d \phi\left(e_{j}\right)\right\rangle \bar{\triangle}^{s+l-1} \tau(\phi) \\
& \left.\left.+\left\langle d \phi\left(e_{j}\right), \bar{\triangle}^{s+l-1} \tau(\phi)\right\rangle \bar{\nabla}_{e_{j}} \bar{\triangle}^{s-l-1} \tau(\phi)\right)\right\} \\
-K\left\{\left\langle\bar{\triangle}^{s-1} \tau(\phi), d \phi\left(e_{i}\right)\right\rangle \bar{\nabla}_{e_{i}} \bar{\triangle}^{s-1} \tau(\phi)\right. & \left.-\left\langle d \phi\left(e_{i}\right), \bar{\nabla}_{e_{i}} \bar{\triangle}^{s-1} \tau(\phi)\right\rangle \bar{\triangle}^{s-1} \tau(\phi)\right\}=0 .
\end{aligned}
$$

By Proposition 3.1 and Lemmas 3.2 and 3.3 , we have

$$
\begin{aligned}
0= & (m K)^{2 s} \tau(\phi)-(m K)^{2 s} \tau(\phi) \\
& -\sum_{l=1}^{s-1}\left\{K\left((m K)^{2 s-2}\|\tau(\phi)\|^{2} \tau(\phi)+(m K)^{2 s-2}\|\tau(\phi)\|^{2} \tau(\phi)\right)\right\} \\
& -K(m K)^{2 s-2}\|\tau(\phi)\|^{2} \tau(\phi) \\
= & -(2 s-1) K(m K)^{2 s-2}\|\tau(\phi)\|^{2} \tau(\phi) .
\end{aligned}
$$

Thus, we have the theorem.

\section{3-HARMONIC MAPS INTO NON-POSITIVE CURVATURE}

In this section we show the non-existence theorem of 3-harmonic maps. G. Y. Jiang showed the following.

Theorem 4.1 ([6]). Assume that $M$ is compact and $N$ is a non-positive curvature, i.e., a Riemannian curvature of $N, K \leq 0$. Then every biharmonic map $\phi: M \rightarrow N$ is harmonic.

We consider this theorem for 3-harmonic maps. First, we recall the following theorem.

Theorem 4.2 ([7]). Let $l=1,2, \cdots$. If $\bar{\triangle}^{l} \tau(\phi)=0$ or $\bar{\nabla}_{e_{i}} \bar{\triangle}^{(l-1)} \tau(\phi)=0$ $(i=1,2, \cdots, m)$, then $\phi: M \rightarrow N$ from a compact Riemannian manifold into a Riemannian manifold is a harmonic map.

Using this theorem, we obtain the next result.

Proposition 4.3. Let $\phi:(M, g) \rightarrow(N, h)$ be an isometric immersion from a compact Riemannian manifold into a Riemannian manifold with non-positive constant sectional curvature $K \leq 0$. Then 3 -harmonic is harmonic.

Proof. Indeed, by computing the Laplacian of the 4-energy density $e_{4}(\phi)$, we have

$$
\begin{aligned}
\triangle e_{4}(\phi)= & \left\|\bar{\nabla}_{e_{i}} \bar{\triangle} \tau(\phi)\right\|^{2}-\left\langle\bar{\triangle}^{2} \tau(\phi), \bar{\triangle} \tau(\phi)\right\rangle \\
= & \left\|\bar{\nabla}_{e_{i}} \bar{\triangle} \tau(\phi)\right\|^{2} \\
& -\left\langle R^{N}\left(\bar{\triangle} \tau(\phi), d \phi\left(e_{i}\right)\right) d \phi\left(e_{i}\right), \bar{\triangle} \tau(\phi)\right\rangle \\
& -\left\langle R^{N}\left(\bar{\nabla}_{e_{i}} \tau(\phi), \tau(\phi)\right) d \phi\left(e_{i}\right), \bar{\triangle} \tau(\phi)\right\rangle,
\end{aligned}
$$


due to the fact that $\phi$ is 3 -harmonic. Here, we consider the right hand side of (9):

$$
\begin{aligned}
&\left\langle R^{N}\left(\bar{\nabla}_{e_{i}} \tau(\phi), \tau(\phi)\right) d \phi\left(e_{i}\right), \bar{\triangle} \tau(\phi)\right\rangle=\left\langleK \left\{\left\langle\tau(\phi), d \phi\left(e_{i}\right)\right\rangle \bar{\nabla}_{e_{i}} \tau(\phi)\right.\right. \\
&\left.\left.-\left\langle d \phi\left(e_{i}\right), \bar{\nabla}_{e_{i}} \tau(\phi)\right\rangle \tau(\phi)\right\}, \bar{\triangle} \tau(\phi)\right\rangle \\
&=K\left\{\|\tau(\phi)\|^{2}\langle\tau(\phi), \bar{\triangle} \tau(\phi)\rangle\right\} .
\end{aligned}
$$

Using Green's theorem, we have

$$
\begin{aligned}
0=\int_{M} \triangle e_{4}(\phi)= & \int_{M}\left\|\bar{\nabla}_{e_{i}} \bar{\triangle} \tau(\phi)\right\|^{2} \\
& -\left\langle R^{N}\left(\bar{\triangle} \tau(\phi), d \phi\left(e_{i}\right)\right) d \phi\left(e_{i}\right), \bar{\triangle} \tau(\phi)\right\rangle \\
& -K\|\tau(\phi)\|^{2}\left\|\bar{\nabla}_{e_{j}} \tau(\phi)\right\|^{2} v_{g} .
\end{aligned}
$$

Then both terms of (10) are non-negative, so we have

$$
\begin{aligned}
0=\triangle e_{4}(\phi)= & \left\|\bar{\nabla}_{e_{i}} \bar{\triangle} \tau(\phi)\right\|^{2} \\
& -\left\langle R^{N}\left(\bar{\triangle} \tau(\phi), d \phi\left(e_{i}\right)\right) d \phi\left(e_{i}\right), \bar{\triangle} \tau(\phi)\right\rangle \\
& -K\|\tau(\phi)\|^{2}\left\|\bar{\nabla}_{e_{j}} \tau(\phi)\right\|^{2} .
\end{aligned}
$$

Especially, we have

$$
\bar{\nabla}_{e_{i}} \bar{\triangle} \tau(\phi)=0
$$

Using Theorem 4.2, we obtain the proposition.

\section{5. $k$-HARMONiC CURVES INTO A EUCLIDEAN SPACE}

In this section, we consider $k$-harmonic curves into a Euclidean space $\mathbb{E}^{n}$ and we give a conjecture. B. Y. Chen [1] defined biharmonic submanifolds of Euclidean spaces.

Definition 5.1 ([1]). Let $x: M \rightarrow \mathbb{E}^{n}$ be an isometric immersion into a Euclidean space. $x: M \rightarrow \mathbb{E}^{n}$ is called a biharmonic submanifold if

$$
\triangle^{2} x=0 \text {, that is, } \triangle H=0,
$$

where $H=-\frac{1}{m} \triangle x$ is the mean curvature vector of the isometric immersion $x$ and $\triangle$ is the Laplacian of $M$.

B. Y. Chen and S. Ishikawa 2] proved that any biharmonic surface in $\mathbb{E}^{3}$ is minimal. Chen [1] also gave a conjecture.

Conjecture 5.2 (1]). The only biharmonic submanifolds in Euclidean spaces are the minimal ones.

There are several results for this conjecture ([5], [3] and [8], etc.). However, the conjecture is still open. I. Dimitric [3] considered a curve case $(m=1)$ and obtained the following theorem.

Theorem 5.3 ([3]). Let $x: C \rightarrow \mathbb{E}^{n}$ be a smooth curve parametrized by arc length, with the mean curvature vector $H$ satisfying $\triangle H=0$. Then the curve is a straight line, i.e., totally geodesic in $\mathbb{E}^{n}$.

We generalize this theorem. First, we define $k$-harmonic submanifolds in Euclidean spaces. 
Definition 5.4. Let $x: M \rightarrow \mathbb{E}^{n}$ be an isometric immersion into a Euclidean space. $x: M \rightarrow \mathbb{E}^{n}$ is called a $k$-harmonic submanifold if

$$
\triangle^{k} x=0, \text { that is, } \triangle^{k-1} H=0(k=1,2, \cdots),
$$

where $H=-\frac{1}{m} \triangle x$ is the mean curvature vector of the isometric immersion $x$ and $\triangle$ is the Laplacian of $M$.

We also consider a curve case $(m=1)$ and obtain the following theorem.

Theorem 5.5. Let $x: C \rightarrow \mathbb{E}^{n}$ be a smooth curve parametrized by arc length, with the mean curvature vector $H$ satisfying $\triangle^{k-1} H=0(k=1,2, \cdots)$. Then the curve is a straight line, i.e., totally geodesic in $\mathbb{E}^{n}$.

Proof. We have $0=\triangle^{k-1} H=-\triangle^{k} x=(-1)^{k+1} \frac{d^{2 k}}{d s^{2 k}} x, k=1,2, \cdots$. Hence $x$ has to be a $(2 k-1)$-th power polynomial in $s$,

$$
x=\frac{1}{2 k-1} a_{2 k-1} s^{2 k-1}+\frac{1}{2 k-2} a_{2 k-2} s^{2 k-2}+\cdots+a_{1} s+a_{0},
$$

where $a_{i}(i=0,1, \cdots, 2 k-1)$ are constant vectors. Since $s$ is the natural parameter we have

$$
\begin{aligned}
1= & \left\langle\frac{d x}{d s}, \frac{d x}{d s}\right\rangle=\left\langle\sum_{i=1}^{2 k-1} a_{i} s^{i-1}, \sum_{i=1}^{2 k-1} a_{i} s^{i-1}\right\rangle \\
= & \left|a_{2 k-1}\right|^{2} s^{4 k-4}+2\left\langle a_{2 k-1}, a_{2 k-2}\right\rangle s^{4 k-5}+\left\{2\left\langle a_{2 k-1}, a_{2 k-3}\right\rangle+\left|a_{2 k-2}\right|^{2}\right\} s^{4 k-6} \\
& \quad \ldots \\
& +\left\{2\left\langle a_{1}, a_{3}\right\rangle+\left|a_{2}\right|^{2}\right\} s^{2}+2\left\langle a_{1}, a_{2}\right\rangle s+\left|a_{1}\right|^{2} .
\end{aligned}
$$

On the right hand side we have a polynomial in $s$, so we must have

$$
a_{2 k-1}=a_{2 k-2}=a_{2 k-3}=a_{2 k-4}=\cdots=a_{3}=a_{2}=0,\left|a_{1}\right|^{2}=1 .
$$

In other words, $x(s)=a_{1} s+a_{0}$ with $\left|a_{1}\right|^{2}=1$, and therefore the curve is a straight line.

Conjecture 5.6. The only k-harmonic submanifolds in Euclidean spaces are the minimal ones.

Especially, when $k=2$, it is the B. Y. Chen conjecture.

\section{REFERENCES}

[1] B. Y. Chen, Some open problems and conjectures on submanfolds of finite type, Soochow J. Math. 17 (1991), 169-188. MR.1143504 (92m:53091)

[2] B. Y. Chen and S. Ishikawa, Biharmonic pseudo-Riemannian submanifolds in pseudoEuclidean spaces, Kyushu J. Math. 52 (1998), no. 1, 167-185. MR1609044 (99b:53078)

[3] I. Dimitric, Submanifolds of $E^{n}$ with harmonic mean curvature vector, Bull. Inst. Math. Acad. Sinica 20 (1992), 53-65. MR1166218 (93g:53087)

[4] J. Eells and L. Lemaire, Selected topics in harmonic maps, CBMS, 50, Amer. Math. Soc., 1983. MR703510 (85g:58030)

[5] T. Ichiyama, J. Inoguchi and H. Urakawa, Bi-harmonic map and bi-Yang-Mills fields, Note di Matematica 28 (2009), 233-275. MR.2640583

[6] G. Y. Jiang, 2-harmonic maps and their first and second variational formulas, Chinese Ann. Math., 7A (1986), 388-402; English translation, Note di Mathematica 28 (2008), 209-232. $\operatorname{MR} 2640582$ 
[7] Sh. Maeta, The second variational formula of the $k$-energy and $k$-harmonic curves, arXiv:1008.3700v1 [math.DG], 22 Aug 2010.

[8] Y.-L. Ou, Some constructions of biharmonic maps and Chen's conjecture on biharmonic hypersurfaces, arXiv:0912.1141v1, [math.DG] 6 Dec 2009.

[9] H. Urakawa, Calculus of variations and harmonic maps, Transl. Math. Monograph. 132, Amer. Math. Soc., 1993. MR1252178 (95c:58050)

[10] S. B. Wang, The first variation formula for K-harmonic mapping, Journal of Nanchang University 13, No. 1, 1989.

Graduate School of Information Sciences, Tohoku University, Aoba 6-3-09 Aramaki Aoba-ku Sendai-Shi Miyagi, 980-8579 JaPAn

Current address: Nakakuki 3-10-9, Oyama-shi, Tochigi, Japan

E-mail address: shun.maeta@gmail.com 